from film solubility, which allows timedependent diffusive interchange between surface and bulk, and acts to reduce the surface tension gradients.

These considerations relate to all waves propagating on water. However, the wind-blown ocean is clearly more complicated. The primary damping effects of surface films are usually found with wavelengths less than about $100 \mathrm{~mm}$, whereas wind-tunnel and sea experiments on wind

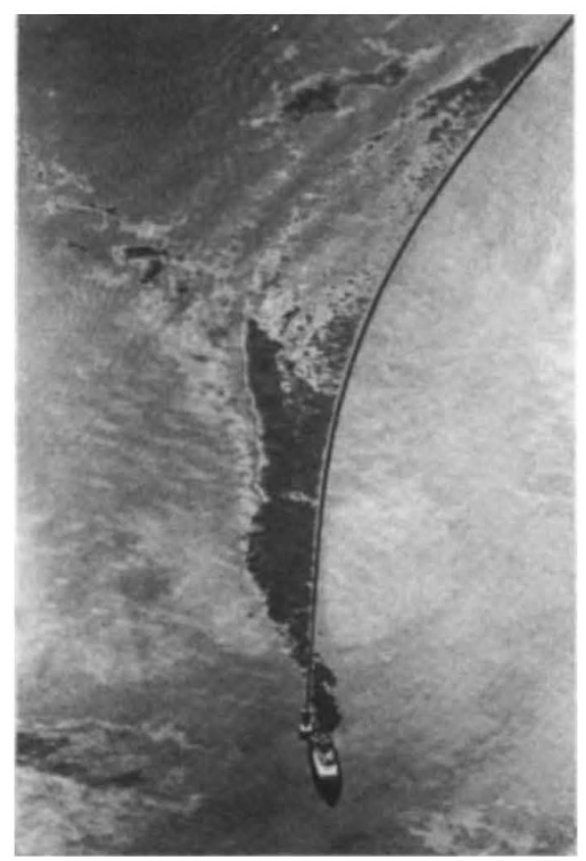

The Exxon Valdez lies grounded in the Prince William Sound, off Alaska. The thick slicks formed by crude oil are less surface active than biogenic slicks, and may be distinguished by analytic techniques.

waves all show very large effects on waves several metres long ${ }^{2}$.

The novel step taken by Alpers and Huhnerfuss is to bring together wavedamping theory with recent results on energy exchange within the surface-wave spectrum, in particular nonlinear wavewave interactions. The authors draw on recent results by Hasselmann and Hasselmann ${ }^{4}$ and Phillips ${ }^{5}$ concerning the distribution of energy within the spectrum and the rate of energy input from the wind.

The mechanism proposed follows the existence of the damping peak in the frequency variation, often near $5 \mathrm{~Hz}$. Wave spectra on film-covered surfaces are duly found to have a pronounced dip at the corresponding wavelength $(50-100$ $\mathrm{mm}$ ). It is proposed that the effect at much longer wavelengths comes from nonlinear wave interactions acting to re-establish the normal equilibrium spectrum, draining energy into the region of the minimum, and away from the longer waves. The authors present approximate calculations which suggest that such a mechanism would indeed explain the observations. They also give preliminary computations in support of the conclusion, although truly quantitative results were not possible because of computer limitations.

The linking of two such complex theoretical areas as film damping and wavewave interactions is not straightforward, and several marginal assumptions have to be made, such as that the nonlinear process itself is not primarily affected by the film, and that other energy 'sinks' such as wave breaking are unaffected. Nevertheless, the results are encouraging when the calculations are compared with laboratory and sea data. The latter include both wave-amplitude and radar backscatter measurements, these being crucially important for the detectability of slicks by real-aperture or synthetic-aperture radars.

Experience of some form of slicks is common. Sun-tan oil can be seen to spread from summer bathers, and on very hot days intricate near-shore patterns of slicks can be seen. These are almost certainly from natural underwater biological activity - fish or plant life - rather than pollution. Even many hundreds of miles from civilization, a calm sunny day will give a 'glassy' sea. Such slicks are frequently unfairly attributed to polluters, and it is therefore vitally important to discriminate slick causes.

Fortunately, major pollutants such as crude oil (see figure), fuel oils and lubricants are usually quite different from biogenic slick materials. They are much less surface active, and they therefore tend to form thicker layers, spreading less. This leads to differences in radar backscatter, and Alpers and Huhnerfuss cite a paper in press on just this topic. There can be even more significant differences in the lidar, thermal infrared and fluorescent ultraviolet signatures of slicks, which could be detected by a multisensor approach, such as that adopted in the United Kingdom by the Warren Spring Laboratory.

Slicks also play an important role in ocean research. Natural slicks have a tendency to become aligned with regions of strong shear, and a remote sensing technique such as imaging radar ${ }^{6}$ or sunglint $^{\dagger}$ can thus provide valuable oceanographic information. The increasing availability of space-borne sensors, such as the radar-imaging satellite ERS-1, will ensure interest in surface films for many years to come.

John C. Scott is in the Ocean Science Division, Admiralty Research Establishment, Portland DT5 2JS, UK.

1. Scott, I C. History of Technology 3, 163-186 (1978).

2. Alpers, W. \& Huhnerfuss, H. J. geophys. Res. 94, 62516265 (1989).

3. Lamb, H. Hydrodynamics, 2nd edn, 552-555 (Cambridge University Press, 1895).

4. Hasselmann, S. \& Hasselmann, W. J. phys. Oceanogr. 15 1369-1377 (1985)

5. Phillips, O.M. J. Fluid Mech. 156, 505-531 (1985).

6. Sheres, D., Kenyon, K.E., Bernstein, R.L. \& Beardsley R.C. J. geophys. Res. 90. 4943-4950 (1985)

R.C. J. geophys. Res. 90. $4943-4950(1985)$.
Scuily-Power, P. Naval Undersea Systems Center Tech nical Document 7611 (26 March 1986)

\section{Flying dust}

MODERN detergents have done much to ease the never-ending problem of keeping things clean. A detergent molecule has an oleophilic end which binds to a greasy dirt particle, and a hydrophilic end which remains solvated by the wash water. So the adhering molecules 'couple' the dirt particles to the water. They lift off the surface to be cleaned, and come into liquid suspension.

Daedalus now points out that exactly the same mechanism could apply in air. Dust is hard to blow off a surface because its particles are poorly coupled to the air. If they could be covered by adsorbed molecules which interacted strongly with the air, they would lift off much more readily. So Daedalus is inventing a gaseous detergent.

$\mathrm{He}$ is synthesizing molecules whose hydrocarbon 'head' readily condenses onto greasy dirt. Their 'tail' is some long, light, flexible chain presenting a big collision cross-section to air molecules, probably a fluorocarbon (hydrocarbon chains, sadly, are oleophilic). Many fluorocarbons are extremely volatile for their molecular weight, and should have a powerful air-lift action on dirt particles.

But the new detergents will not be a gas. For by analogy with water-based detergents, it should work best at concentrations around 0.1 per cent, or a partial pressure of 0.001 atmospheres. And since it must condense out of the air and be adsorbed hygroscopically on the dirt at this concentration, this must be its saturated-vapour pressure at room temperature: implying a boilingpoint around $180^{\circ} \mathrm{C}$. So 'Aerosolve' (as it will be called) will have quite a high molecular weight, with room for much chemical subtlety.

Its first mass application will be as a vacuum-cleaning aid, wafted ahead of the machine by an evaporator on its cleaning head. The stickiest dust will lost its grip on the carpet and be effortlessly sucked up. More radically, a room permanently saturated with Aerosolve would never get dirty in the first place, for the dust would stay harmlessly in air-suspension. Just hang a wick or paper strip saturated with Aerosolve in the room, and your cleaning problems are solved. The gradual insidious dirtying of all surfaces, which only becomes apparent when you rub a finger over them, or take a picture down and find a clean patch behind it, would simply not happen. The slow fouling of air-cooled laboratory equipment, which makes the servicing of old computers, and so on, such a grimy experience, could similarly be prevented.

Even better, Aerosolve vapour in the air should prevent dust and bacteria being deposited in the lungs as well. The benevolent detergent-vapour could thus help to prevent pneumonia, asbestosis, and some of the nasty diseases that afflict smokers.

David Jones 